\title{
Sequential segmentectomy: Known knowns, known unknowns, and unknown unknowns
}

\author{
Ze-Rui Zhao, MD, and Calvin S. H. Ng, MD, FRCS
}

\footnotetext{
From the Division of Cardiothoracic Surgery, Department of Surgery, The Chinese University of Hong Kong, Prince of Wales Hospital, Hong Kong SAR, China.

Disclosures: Z.-R.Z. declares no conflicts of interest. C.S.H.N. has an electromagnetic navigation bronchoscopy on loan from Medtronic Inc.

Received for publication Nov 1, 2017; accepted for publication Nov 15, 2017; available ahead of print Dec 6, 2017.

Address for reprints: Calvin S. H. Ng, MD, FRCS, Division of Cardiothoracic Surgery, The Chinese University of Hong Kong, Prince of Wales Hospital, Shatin, N.T., Hong Kong SAR, China (E-mail: calvinng@surgery.cuhk. edu.hk).

J Thorac Cardiovasc Surg 2018;155:1305-6

$0022-5223 / \$ 36.00$

Copyright (C) 2017 by The American Association for Thoracic Surgery

https://doi.org/10.1016/j.jtcvs.2017.11.061
}

Anatomic segmentectomy is increasingly advocated for treating select cases of early-stage non-small cell lung cancer $^{1}$ or lung metastases deep in the parenchyma. In this issue of the Journal, Shimizu and colleagues ${ }^{2}$ from Gunma University Hospital, Japan, describe the first case of sequential superior segmentectomy (S6) in the left lower lobe in a patient who previously underwent left posterior basilar segmentectomy (S10).

In this case of a patient with limited lung function because of prior right upper lobectomy, right lower lobe tri-segmentectomy, and left S10 segmentectomy, the forced expiratory volume of 1.0 remained comparable before and after the left S6 segmentectomy. Better lung function preservation is the theoretic advantage of segmentectomy over lobectomy. However, Nomori and colleagues ${ }^{3}$ showed that this only occurs when no more than 2 segments are resected. Furthermore, data on the effects of repeat segmentectomy on pulmonary function in patients with prior major lung resections are lacking. The potential explanation for "unchanged" lung function in the current patient could be due to the removal of mostly nonfunctional granuloma scar in S6 or the compensation of the remaining parenchyma. Thus, the previous resection of left $\mathrm{S} 10$ instead of bi- or tri-segmentectomy of the basilar region is important for enabling the patient to receive the left S6 resection. An additional consideration for same lobe sequential segmentectomy with previous lateral (S9) or posterior basal (S10) segmentectomy is the prior dissection of the deeply located segmental artery and bronchus, which can cause great technical difficulties for future mobilization. A novel technique by using a posterior approach for these segments ${ }^{4}$ can avoid manipulation from the oblique fissure, which may facilitate segmental structure division if sequential superior segmentectomy (S6) is needed.

To achieve an oncologically sound resection margin during segmentectomy, rigorous preoperative 3-dimensional simulation is recommended. The lesion location may

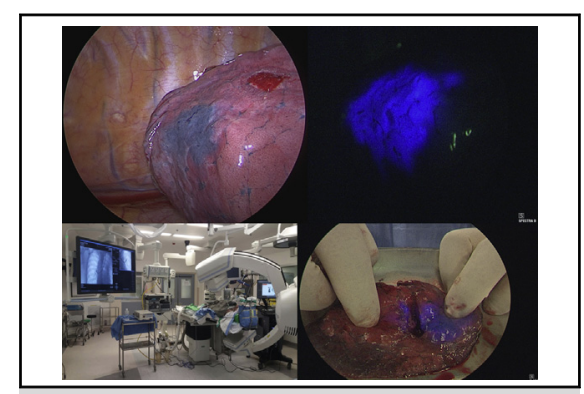

Electromagnetic navigation bronchoscopy methylene blue indocyanine green dual marking in the hybrid operating room for lesion localization and margin.

\section{Central Message}

Sequential segmentectomy data on its benefits and best approach are lacking. Preoperative planning, special techniques for localization, and delineating the resection margin may improve surgical precision.

See Article page 1302 .

warrant complex segmentectomy or necessitate additional wedge resection of adjacent segments. Another important consideration is the intraoperative localization of the small lung lesion and identification of inter-segmental plane for placing the staplers. Currently, the inflation-deflation technique of the target segmental bronchus is probably the most widely used method for defining the segment. We believe the use of electromagnetic navigation bronchoscopy indocyanine green dye marking localization of the lesion and indocyanine green intravascular injection in the hybrid theatre ${ }^{5}$ may not only enable accurate lesion localization and enhanced visualization of inter-segmental lines but also help to assess the resection margins via intraoperative cone-beam computed tomography for higher surgical precision (Figure 1). Such preoperative planning and specialized intraoperative techniques may be even more relevant for sequential segmentectomy with rotated and distorted anatomy.

We congratulate the authors on well-performed sequential segmentectomies in this select patient. The ability to perform sequential segmentectomy whether on the ipsilateral side or the same lobe with previous segmentectomy may become increasingly important as an increasing number of early lung cancers are detected through screening programs and advances in targeted therapy continue to change the course of cancer 


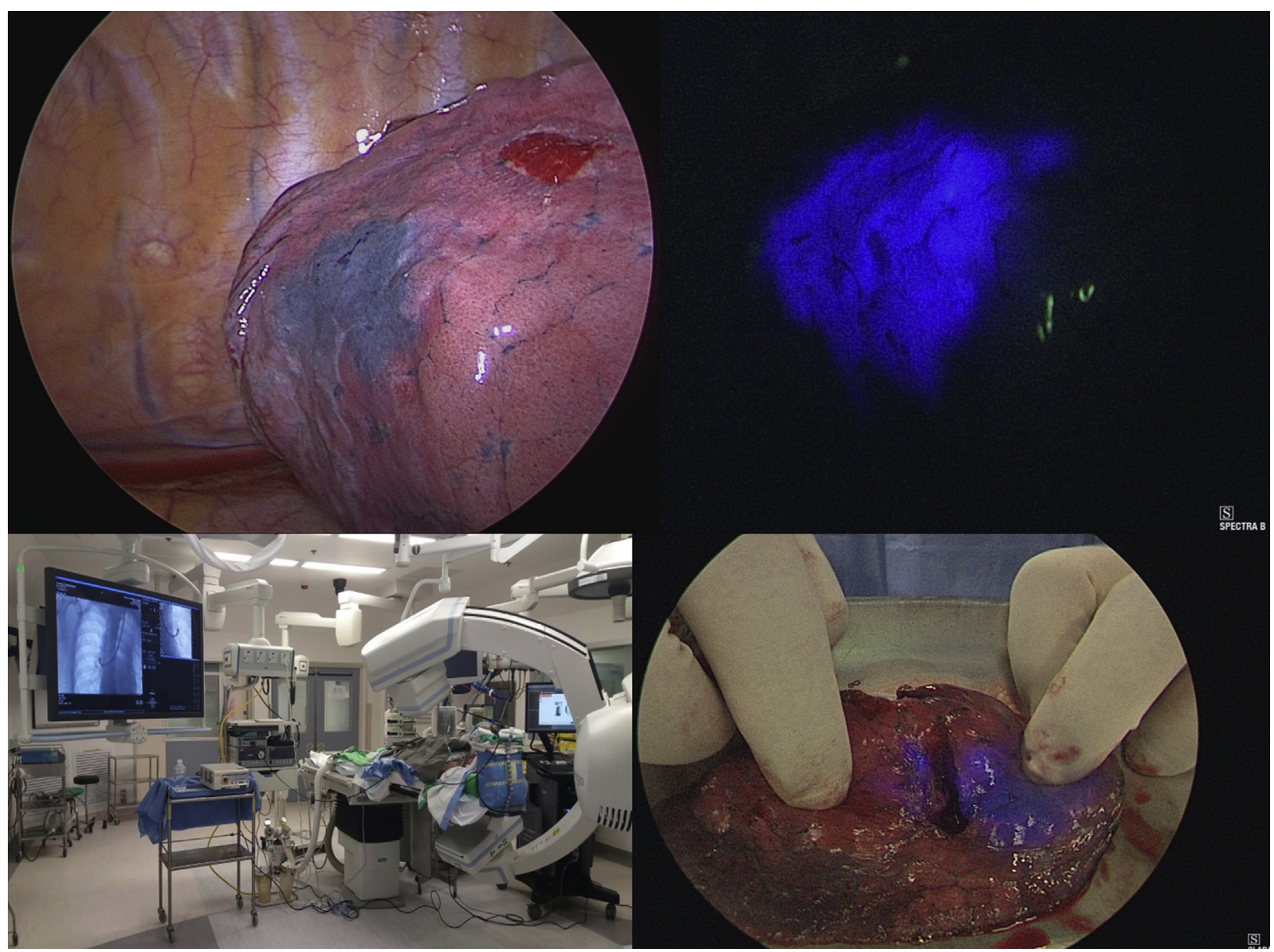

FIGURE 1. Electromagnetic navigation bronchoscopy methylene blue indocyanine green dual marking in the hybrid operating room for lesion localization and margin.

management. Evidence is lacking in this re-segmentectomy cohort, particularly with regard to pulmonary function preservation and optimizing surgical intervention for sequential resections.

\section{References}

1. Zhao ZR, Situ DR, Lau RWH, Mok TSK, Chen GG, Underwood MJ, et al. Comparison of segmentectomy and lobectomy in stage IA adenocarcinomas. J Thorac Oncol. 2017;12:890-6.
2. Shimizu K, Nakazawa S, Mogi A, Kuwano H. Segmentectomy of the left superior segment (S6) four years after segmentectomy of the left dorsal-basal segment (S10). J Thorac Cardiovasc Surg. 2018;155:1302-4.

3. Nomori H, Cong Y, Sugimura H. Systemic and regional pulmonary function after segmentectomy. J Thorac Cardiovasc Surg. 2016;152:747-53.

4. Endoh M, Oizumi H, Kato H, Suzuki J, Watarai H, Masaoka T, et al. Posterior approach to thoracoscopic pulmonary segmentectomy of the dorsal basal segment: a single-institute retrospective review. J Thorac Cardiovasc Surg. 2017:154:1432-9.

5. Zhao ZR, Lau RWH, Ng CSH. Hybrid theater and uniportal video-assisted thoracic surgery: the perfect match for lung nodule localization. Thorac Surg Clin. 2017;27:347-55. 